\title{
Modelling Turbine Vibration in Terms if its Load Variation
}

\author{
DR. T. J. CHALKO \\ University of Melbourne, Department of Mechanical and Manufacturing Engineering, Parkville 3052, Victoria, Australia \\ Scientific Engineering Research, 29 Hotham St. East St. Kilda, 3183 Melbourne, Victoria, Australia
}

DR. D. X. LI

Scientific Engineering Research, 29 Hotham St. East St. Kilda, 3183 Melbourne, Victoria, Australia

\begin{abstract}
This paper is based on a case history of a $650 \mathrm{MW}$ turbine-generator, which changed its vibration significantly every time when a change of its thermal/electrical load was made. Significant changes of vibration amplitude and phase distribution along the shaft indicated the contribution of different modes of vibration. Interestingly, vibration of other identical units manufactured by the same manufacturer were not sensitive to load variation. A vibration monitoring system, relying on observing slow trends in vibration data, was not able to interpret the significant vibration changes. In particular, it was not clear, whether or not there was a fault in the system and whether or not the unit was safe to operate. The paper presents problem modelling, analysis and the explanation for vibration changes. Presented analysis explains that vibration changes were not associated with the fault in the system but they were a natural response of the system to parameter change.
\end{abstract}

Key Words: Rotordynamics; Turbine; Turbogenerator; Alignment; Vibration

\section{INTRODUCTION}

$\mathbf{M}$ odelling turbogenerator vibration in terms of its load requires identification of system parameters which undergo changes when a turbine load is changed. In this study, changes in the rotor-bearing-foundation system temperature distribution and the rotor alignment were considered. While the temperature distribution change and corresponding change in material properties seems obvious, the alignment change requires some explanation.

It is a verified fact, that the alignment of a multibearing turbogenerator set changes as a function of its operating conditions. In particular, alignment at full turbine load ("hot"), may differ significantly from the alignment when turbine is not loaded ("cold"). For example, investigations by Webster and Gibson [1977] and Hashemi [1983] have shown substantial changes in alignment arising from turbine operating conditions. Most turbine manufacturers have experimentally verified "cold" and "hot" alignments for their turbines.
In a multi-bearing rotor system, any change in alignment causes changes in all bearing reaction distribution. This is due to the fact that a multi-bearing rotor system is hyperstatic (not statically determinate). In a large turbine-generator set, rotor is usually supported by hydrodynamic bearings, which have nonlinear characteristics. In such system, a change in hydrodynamic bearing reactions causes changes in bearing equilibrium positions and consequently the bearing dynamic characteristics (stiffness and damping properties). This change in bearing dynamic properties changes dynamic characteristic of the entire system, influencing the system response and stability. A number of researchers have presented important results, demonstrating the influence of bearing transverse shifts (alignment) in rotor dynamics. For example, the effects of bearing shifts on rotor stability threshold were analysed by Mayes and Davies [1982], Krodkiewski et al. [1983]. Hori and Uematsu [1980] obtained bearing eccentricity maps for various stability thresholds. Hashemi [1983] demonstrated the effects of bearing misalignment on the bearing load distribution, 
journal eccentricity in equilibrium, stability of the system, synchronous and subsynchroneous response of a multi-bearing rotor system. Parszewski and Krodkiewski [1986] introduced alignment parameters as independent variables, enabling dynamic analysis such as unbalance response or stability analysis to be performed as a function of machine alignment (in the machine configuration domain). $\mathrm{Li}$ [1990] also made important contribution to various aspects of analysis of linearized multibearing systems in the alignment domain. Parszewski, Chalko and Li [1988] considered optimisation of turbine alignment from the point of view of minimisation of system vibration at nominal speed and presented calculations for $200 \mathrm{MW}$ turbo-generator.

In view of the above, it becomes clear that the formulation of the turbine model should include all bearing and shaft alignment parameters.

Available commercial computational algorithms do not provide efficient tools to develop turbine models in terms of bearing and shaft alignment. The method presented below and its numerical implementation on a Pentium ${ }^{\circledR}$ microcomputer (Chalko and Li [1993, 1994]) gives engineers a practical, efficient and comprehensive turbine modelling tool, enabling them to study various aspects of turbine alignment and its effects on turbine vibration. An example $650 \mathrm{MW}, 8$ bearing turbine and its mathematical model will be used to explain the method and analyse vibration changes due to the thermal load variation of the turbine.

\section{TURBOGENERATOR MODELLING}

The turbogenerator modelling technique was described by authors $[1988,1993,1994]$ but it is summarised here for clarity. In the method, a global model for the turbogenerator system is synthesised from 3 main substructures: rotor system, foundation-casings system and journal bearings.

\section{Rotor modelling}

Rotor substructure model is formulated using the FEM technique. Initial several hundred elements (see Fig. 1) were condensed in two condensation stages to give a 25 super element substructure, having 26 nodes, shown in Fig. 1.

Rotor substructure is characterised by 2 sets of stiffness and mass matrices, one for static calculations $\left[K_{\mathrm{r}}\right]_{\mathrm{s}}$ and $\left[M_{\mathrm{r}}\right]_{\mathrm{s}}$ and the second for dynamic calculations $\left[K_{\mathrm{r}}\right]_{\mathrm{d}}$ and $\left[K_{\mathrm{r}}\right]_{\mathrm{d}}$. For each turbine load, the rotor subsystem experiences different temperature distribution. Therefore, for each temperature distribution ("cold"-light turbine

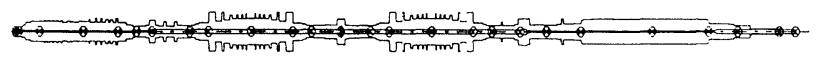

FIGURE 1 Model of the turbine rotor.

load and "hot"-full turbine load) a separate rotor substructure was formulated.

\section{Foundation and casings modelling}

Foundation substructure is characterised by mass, stiffness and damping matrices along the required set of connecting coordinates. Such substructure could be obtained using a general purpose FEM program. The foundation-casings substructure presented here was generated using ANSYS ${ }^{\circledR}$ FEM program and a combination of solid and plate elements. The model is illustrated in Fig. 2. Reduction of mass and stiffness matrices was performed, to obtain condensed mass and stiffness matrices of the structure $\left[M_{\mathrm{f}}\right]$ and $\left[K_{\mathrm{f}}\right]$ along required set of coordinates. The foundation damping matrix $\left[D_{\mathrm{f}}\right]$ was calculated by determining damping ratios for modes of the foundation substructure.

\section{Bearing modelling}

The considered system had 8 hydrodynamic bearings: bearing 1 and 2 had 6 rolling pads each, preloaded; other bearings had multiple fixed elliptical pads. Each bearing geometry was different. A detailed description of bearing characteristics calculation (see for example Li [1990]) is beyond the scope of this article. However, a brief description of the algorithms with references to source literature is given below. The optimised Finite Difference Method was used to solve Reynolds Equation with appropriate boundary conditions, and approximate thermal

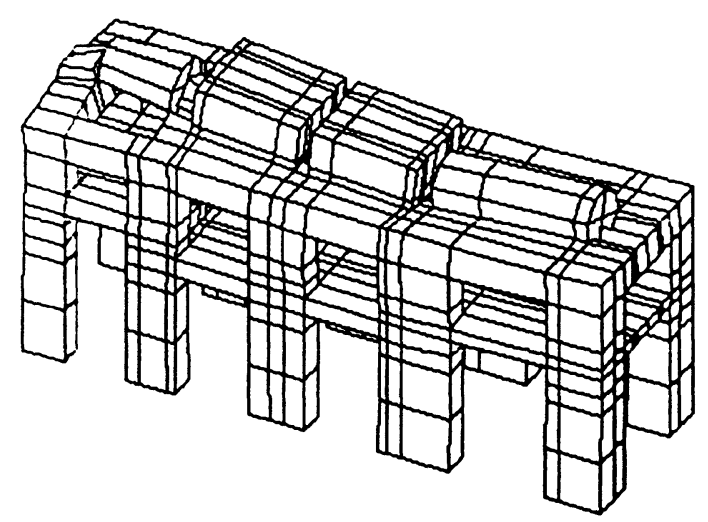

FIGURE 2 Foundation—casings substructure. 
effects for each bearing pad to determine the oil pressure on each pad. The calculation was performed in 2 dimensional grid, giving circumferential as well as longitudinal pressure distributions. Bearing total hydrodynamic force was then calculated by integrating obtained pressures from all pads. The hydrodynamic bearing force vector $\mathbf{f}_{h}$ is a function of journal position and velocity in the bearing as well as the journal angular velocity $\Omega$ :

$$
\mathbf{f}_{h}=\mathbf{f}_{h}\left(x_{J B}, y_{J B}, \dot{x}_{J B}, \dot{y}_{J B}, \Omega\right)
$$

The Newton iteration method (after Wang et al [1979]) was used to determine the shaft equilibrium in each bearing, given the bearing load (bearing reaction). For each rolling pad, an additional iteration loop was performed, searching for the rolling pad equilibrium. After the bearing equilibrium was found, the linearized stiffness and damping matrices were calculated. It should be pointed out that bearing linearization was performed using a global bearing hydrodynamic force calculation.

Bearing equilibrium positions and linearized bearing stiffness and damping matrices were calculated each time the shaft alignment, rotating speed or any substructure property was changed. This ensured maximum possible accuracy of the linearized turbine model.

\section{Alignment modelling}

For the analysis presented further in this paper, the turbine alignment was specified by given bearing pedestal positions. Since a multi-bearing rotor system is hyperstatic (not statically determinate) and hydrodynamic bearings characteristics are nonlinear, an iterative method was applied to find global system equilibrium configuration (alignment) given all bearing pedestal positions. For the equilibrium configuration obtained, the required linearized bearing stiffness and damping matrices were calculated (Parszewski et al. [1988], Chalko and Li [1993, 1994]).

\section{Global system synthesis from substructures}

After linearized bearing stiffness and damping matrices were calculated, all substructures become linear, and the formulation of the global mass $[M]$, damping $[D]$ and stiffness $[K]$ matrices for the entire turbogenerator system becomes straightforward (Parszewski et al [1986, 1988]). The linearized equation of motion for the entire system could be therefore written in the form:

$$
[M]\{\ddot{q}\}+[D]\{\dot{q}\}+[K]\{q\}=\{F(t)\}
$$

where $\{\mathrm{q}\}=\left[\mathrm{q}_{\mathrm{r}}, \mathrm{q}_{\mathrm{f}}\right]^{\mathrm{T}}$ is a generalised coordinate vector, containing $\mathrm{n}$ displacement coordinates of all rotor $\left\{\mathrm{q}_{\mathrm{r}}\right\}$ and foundation $\left\{\mathrm{q}_{\mathrm{f}}\right\}$ nodes along considered directions.

\section{CALCULATION RESULTS}

To analyse turbine vibration changes due to load variation, results of modelling and calculations for 2 turbine models, corresponding to 2 load cases will be compared and discussed further in detail:

Case 1: turbine with partial load, referred further as "cold", due to lower shaft temperatures.

Case 2: turbine with full nominal load, referred further as "hot".

The system response to 3 different unbalance distribution will be analysed:

Unbalance 1: $0.05 \mathrm{kgm}$ located at the middle balancing plane of LP1 stage

Unbalance 2: $0.05 \mathrm{kgm}$ located at the balancing plane of LP1 stage near bearing 3

Unbalance 3: $0.05 \mathrm{kgm}$ located at the balancing plane of LP1 stage near bearing 4

As the turbine manufacturer suggested, "hot" alignment was considered different from "cold" alignment mainly at the first 2 bearings of the turbine (at the high pressure stage). Estimated (smallest) differences in the alignment in the vertical direction did not exceed $0.8 \mathrm{~mm}$ and in the horizontal direction were less than $0.2 \mathrm{~mm}$. The assumed shaft alignments for "cold" and "hot" turbine, suggested by the turbine manufacturer, are presented in Fig. 3 and Fig. 4 respectively.

Fig. 3 presents the calculated shaft unbalance response at the nominal speed 3600 RPM for the "cold" turbine as well as the corresponding shaft alignment. In Fig. 4 similar plots for "hot" turbine are presented. Comparison of shaft unbalance responses shown in Fig. 3 and Fig. 4 indicates visible differences in rotor vibration amplitude and phase distribution. It can be seen, that vibration

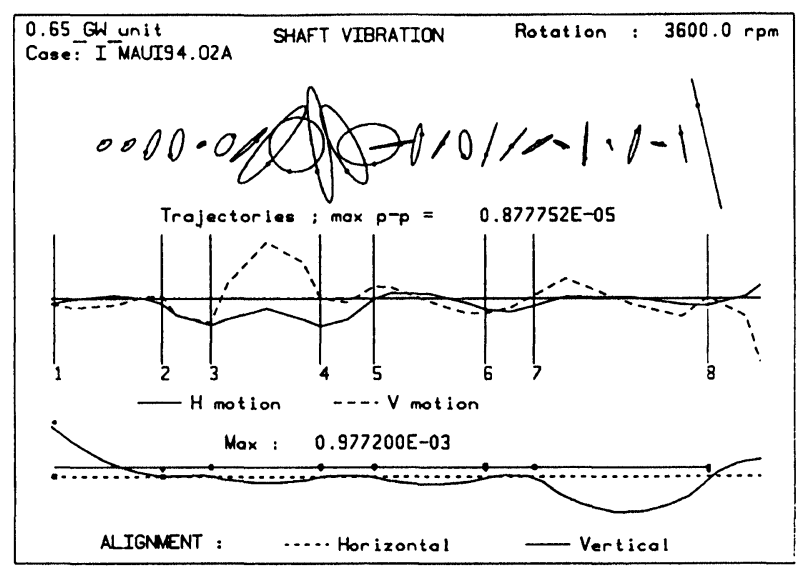

FIGURE 3 Unbalance 1 response: "cold" rotor trajectories and the corresponding alignment. 


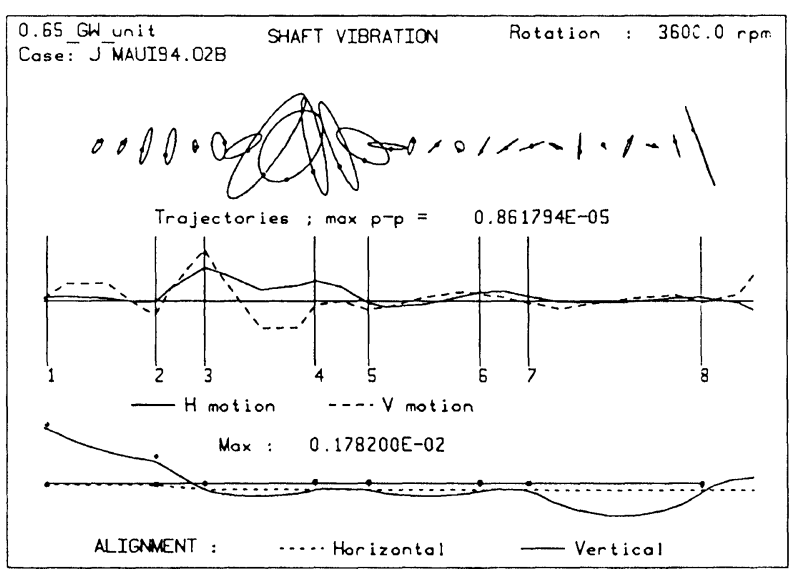

FIGURE 4 Unbalance 1 response: "hot" rotor trajectories and the corresponding alignment.

changes are most significant at bearings 3 and 4 (Low Pressure stage 1).

Plots in Fig. 5 and Fig. 6 indicate quite dramatic changes in vibration due to the turbine load change. For example at 3600 RPM (the nominal speed), amplitude increases for the "hot" system reach $100 \%$ of the initial "cold" system amplitude at bearing 3 (Fig. 5). Dashed lines with no labels show the system response when only rotor temperatures were changed. This result indicates, that the calculated response change is due mainly to change in the system alignment. Unbalance response of the system was calculated for other unbalance distributions and results are presented in Fig. 7 and Fig. 8 for unbalance 2 and 3 respectively. Only vibration at bearings 3 and 4 are shown.

Above results show not only different responses for different unbalance distributions (it was expected), but

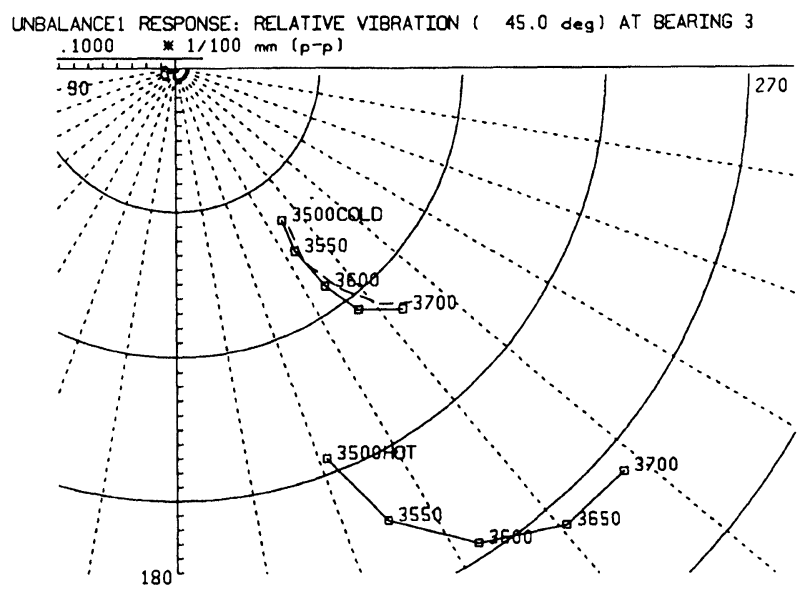

FIGURE 5 Unbalance 1 response: relative shaft vibration for "cold" and "hot" turbine at bearing 3 .

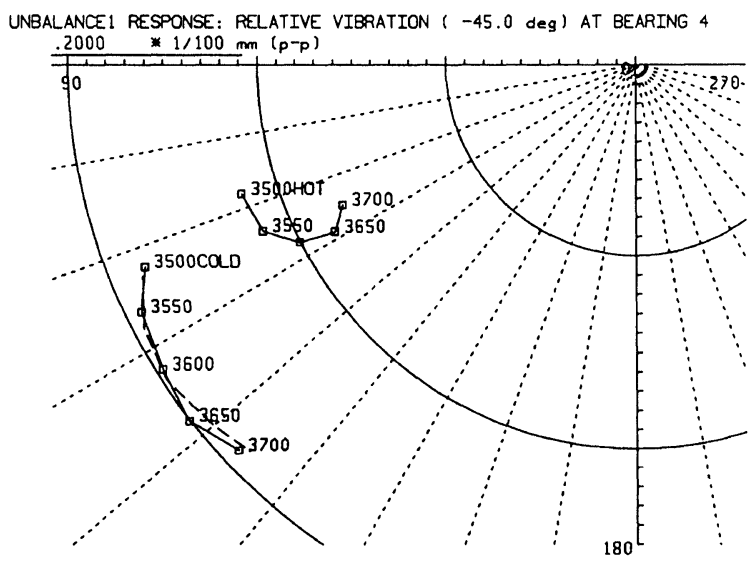

FIGURE 6 Unbalance 1 response: relative shaft vibration for "cold" and "hot" turbine at bearing 4.

most interestingly indicate, that the system vibration sensitivity to load changes depends strongly on the rotor unbalance. Such a relationship could explain, why vibrations of otherwise identical turbines may show different sensitivity to load changes.

To analyse reasons for the above sensitivity an eigenvalue analysis was performed for the linearized system (2) (Chalko and Li [1994]).

\section{EIGENVALUE ANALYSIS}

Matrices $[D]$ and $[K]$ in the equation (2) are not symmetric, due to the fact, that all journal bearings in the system have non symmetric stiffness and damping matrices. For such systems, an eigenvalue analysis requires calculation of 2 sets of different eigenvectors. called right

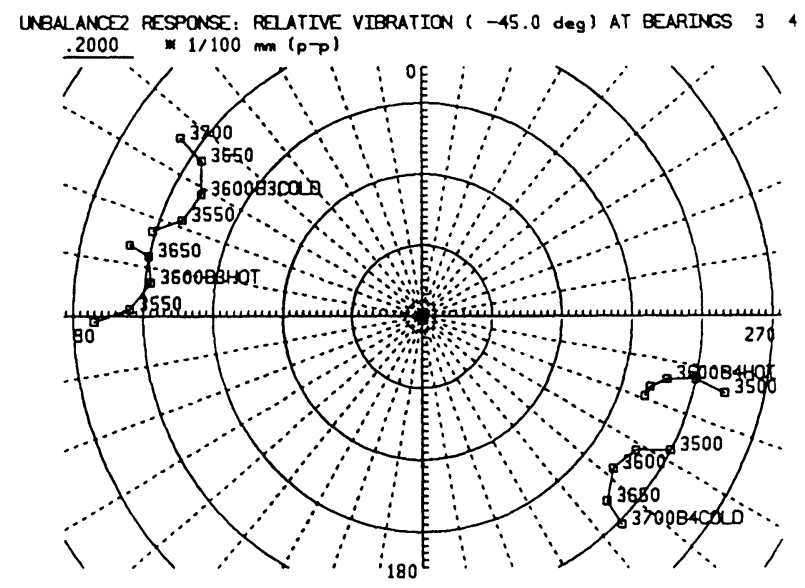

FIGURE 7 Unbalance 2 response: relative vibration for "cold" and "hot" turbine at bearings 3 and 4 . 


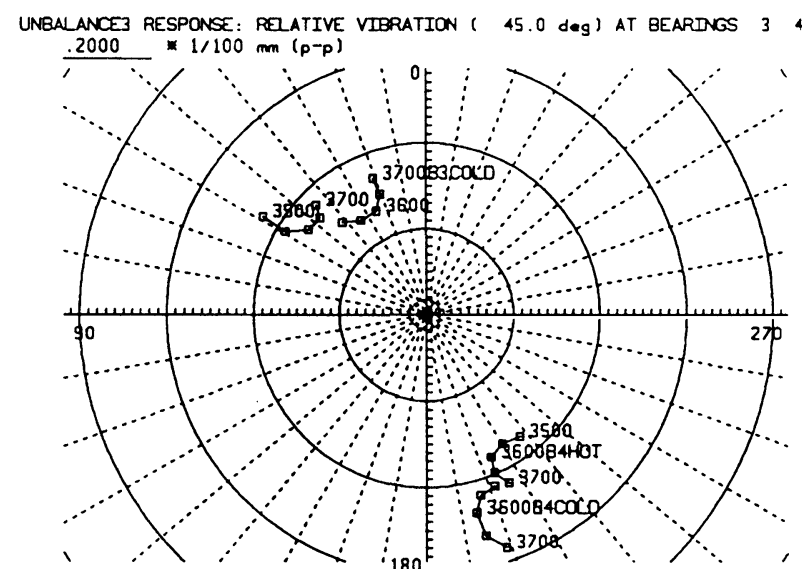

FIGURE 8 Unbalance 3 response: relative vibration for "cold" and "hot" turbine at bearings 3 and 4 .

and left eigenvectors. In particular, solving the eigen value problem for the matrix (3)

$$
\left[E_{R}\right]=-[A]^{-1}[B]
$$

will give complex eigenvalues $\lambda_{j}=\lambda_{R}+i \lambda_{j l} ; \mathrm{j}=1 . .2 \mathrm{n}$ and corresponding set of right complex eigenvectors $\left\{Q_{R}\right\}_{j}, \mathrm{j}=1 . .2 \mathrm{n}$, while solving the eigenvalue problem for the matrix (4)

$$
\left[E_{L}\right]=-\left[[B][A]^{-1}\right]^{T}
$$

will result in identical eigenvalues and a corresponding set of left complex eigenvectors $\left\{Q_{L}\right\}_{j}, \mathrm{j}=1 . .2 \mathrm{n}$. Matrices $[A]$ and $[B]$ are defined as follows:

$$
\begin{aligned}
& {[A]=\left[\begin{array}{cc}
0 & {[M]} \\
{[M]} & {[D]}
\end{array}\right]} \\
& {[B]=\left[\begin{array}{cc}
-[M] & 0 \\
0 & {[K]}
\end{array}\right]}
\end{aligned}
$$

\section{Interpretation of left and right eigenvectors}

Physical meaning of left and right eigenvectors could be explained by expressing the harmonic response of the system in terms of eigenvalues and eigenvectors. Consider the response of the system (2) to the unbalance excitation of the form (7):

$$
\{F(t)\}=\{U\} \Omega^{2} e^{\Omega}
$$

System complex response vector $\{\mathrm{q}\}$ expressed in terms of a sum of modal response vectors $\{Q\}_{j}$

$$
\{q\}=\sum_{j=1}^{n_{y}}\{Q\}_{j} e^{i \Omega t}
$$

where the $\mathrm{j}$-th modal response vector $\{Q\}_{j}$ is:

$$
\{Q\}_{j}=\frac{a_{j}\left\{Q_{R}\right\}_{j}}{-\gamma_{R j}+i\left(\Omega-\gamma_{i j}\right)}+\frac{b_{j}\left\{\bar{Q}_{R}\right\}_{j}}{-\gamma_{R j}+i\left(\Omega+\gamma_{i j}\right)}
$$

Equation (9) shows, that the system response is composed from complex conjugate pairs of right eigenvectors $\left\{Q_{R}\right\}_{j}$ and $\left\{\bar{Q}_{R}\right\}_{j}$. Complex coefficients $a_{j}$ and $b_{j}$ are modal excitation factors, expressed in terms of the system unbalance distribution $\{U\}$ and complex conjugate pairs of left eigenvectors $\left\{Q_{L}\right\}_{j}$ and $\left\{\bar{Q}_{\mathrm{L}}\right\}_{j}$ :

$$
\begin{gathered}
a_{j}=\left\{Q_{L}\right\}_{j}^{T}\{U\} \Omega^{2} \\
b_{j}=\left\{\bar{Q}_{L}\right\}_{j}^{T}\{U\} \Omega^{2}
\end{gathered}
$$

From (9) and (10) it can be seen that left eigenvectors are modal weighting factors for the unbalance distribution $\{U\}$.

Eigenvalues were calculated for "cold" and "hot" systems and used to create a map of critical speeds presented in Fig. 9. Significant shifts of eigenvalues 26 and 27 between "cold" and "hot" operating conditions are shown. In particular, it can be seen, that for the "cold" system the eigenvalue 26 is closer to $3600 \mathrm{rpm}$ (the nominal speed), while for the "hot" system-eigenvalue 27 is closer to $3600 \mathrm{rpm}$. Indeed the right complex eigenvector 26 for "cold" system at $3600 \mathrm{rpm}$, shown in Fig. 10 is quite similar to the system unbalance response presented in Fig. 3, especially in vertical direction.

By analysing shapes of eigenvectors 26 and 27, shown in Fig. 10-13, it becomes clear why the system vibration

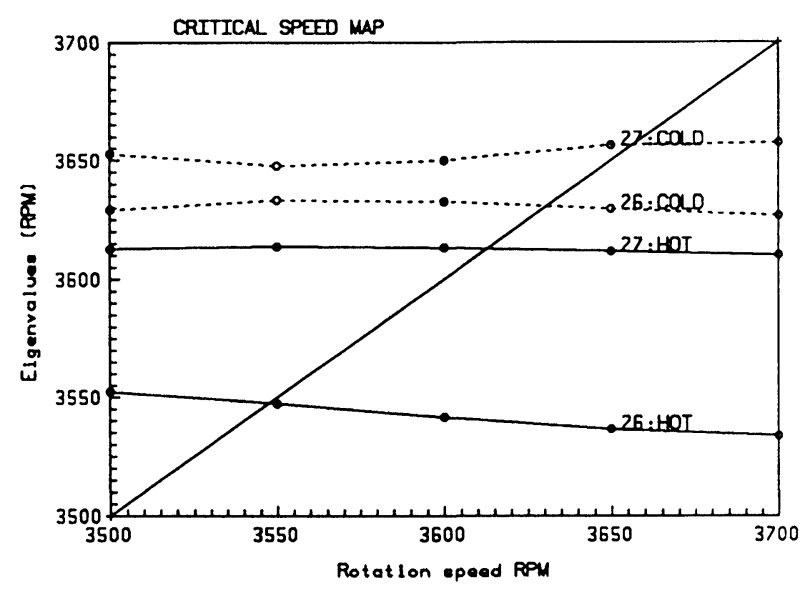

FIGURE 9 Eigenvalues 26 and 27 for "cold" and "hot" system. 


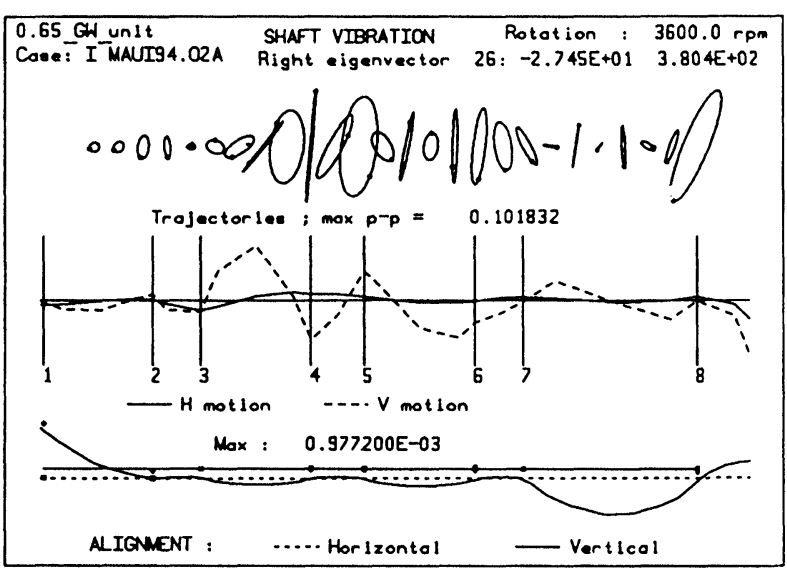

FIGURE 10 Right eigenvector 26 for "cold" system.

sensitivity to load changes depends strongly on the rotor unbalance as we have seen in Fig.5, 6, 7, and 8.

The left eigenvector 26 (Fig. 11) has maximum trajectory at the mid-span of the LP1 turbine and corresponding trajectories at other balancing planes are much smaller. The left eigenvector 27 (Fig. 13), on the other hand has a minimum trajectory node at the mid-span of LP1, and large trajectories at two other balancing planes of LP1.

If the unbalance is located at the mid span (unbalance 1), the system shows high sensitivity, because the right eigenvector 26 (Fig. 10) contribution to the system response remains high, even if the eigenvalue 26 is shifted away from the resonance (see Fig. 9).

If the unbalance is located at either side balancing plane of LP1 (unbalance 2 and 3), the system is less sensitive, because the right eigenvector 26 contribution is significantly reduced due to the shape of the left eigenvector 26 (unbalance is applied close to node) and the

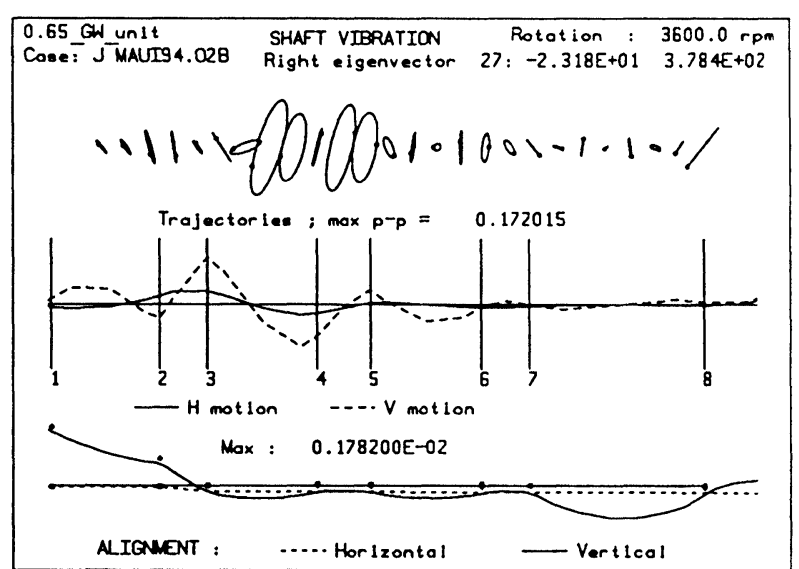

FIGURE 12 Right eigenvector 27 for "hot" system.

fact that the corresponding eigenvalue is shifted away from the resonance (see Fig. 9).

\section{PRACTICAL IMPORTANCE}

1. Reasons for significant vibration changes related to the thermal load changes of the turbine were explained. It was found, that vibration changes are mainly due to the change in the system alignment, rather than due to the temperature related changes of material properties.

2. Presented analysis explains, that significant vibration changes due to load variation were not associated with the fault in the system but they were a natural response of the system to the alignment change, caused by the thermal load variations.

3. It was explained, why the two otherwise identical turbines could show different sensitivity to changes in thermal load and alignment.

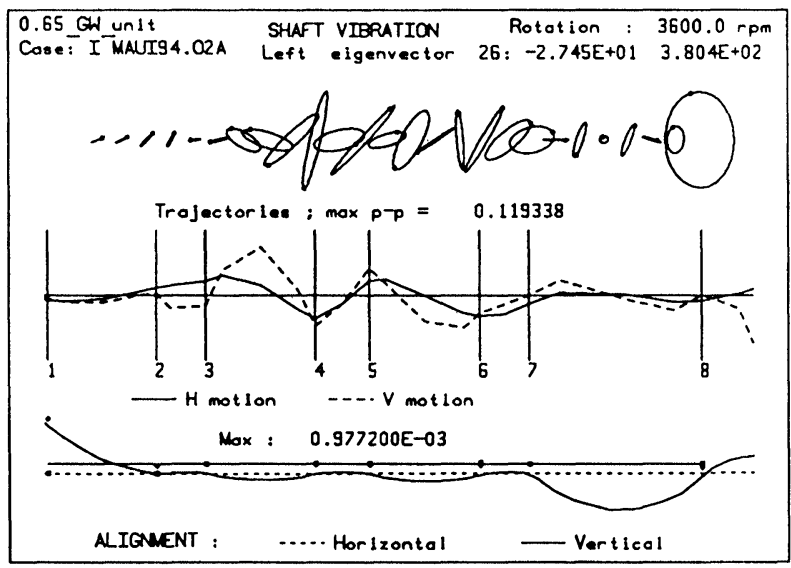

FIGURE 11 Left eigenvector 26 for "cold" system.

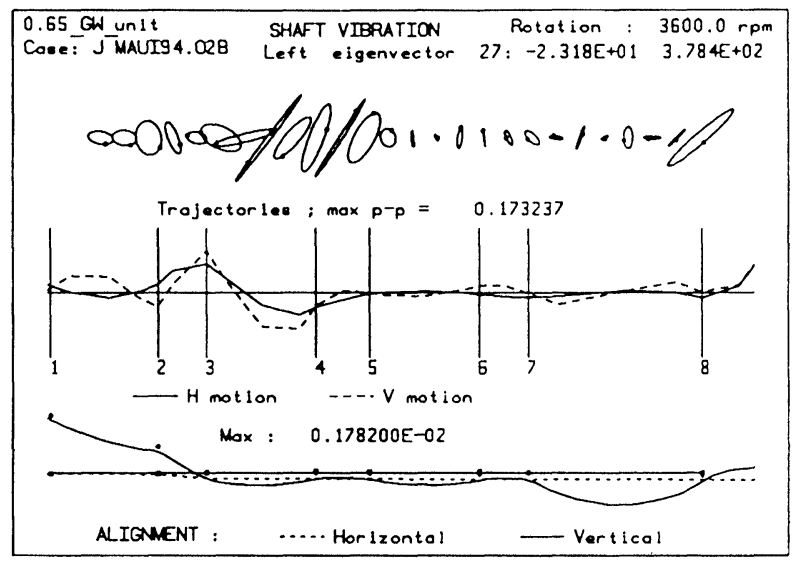

FIGURE 13 Left eigenvector 27 for "hot" system. 
4. The method enables to predict the system sensitivity to alignment changes and gives a simple remedy to reduce such sensitivity if required, without any structural changes of the machine.

\section{CONCLUSIONS}

The results demonstrate, that presented method is suitable for modelling and analysis of turbine vibration in terms of its load, providing that the corresponding alignment changes are known. The following conclusions arise from above analysis:

1. Small horizontal and vertical alignment changes (caused by thermal load changes etc. ) could cause significant changes in vibration of the large multibearing turbine

2. Vibration changes due to turbine load variation occur mainly due to changes in the system alignment. Effects of different temperature distributions along the shaft and related changes in material properties are negligible. Accuracy of vibration modelling depends therefore on the accuracy of alignment variation estimates. In this study, experimentally confirmed estimates of "hot" and "cold" alignments were used.

3. System sensitivity to load changes may depend strongly on the residual unbalance distribution. This explains, why the two otherwise identical turbines could show different sensitivity to changes in load and alignment.

4. A specific unbalance distribution (expressed in terms of rotor balancing planes) could be found, for which the system vibration is the most sensitive to the turbine load changes. It was shown, that the same system with different unbalance distribution could be much less sensitive to load changes.

5. The remedy to reduce the system sensitivity to alignment changes, caused by the thermal load or any other reason is to change the residual unbalance distribution. This is a very practical method, because it does not require any structural changes of the machine. The most effective residual unbalance change could be found using the numerical model of the turbine described in this article.

\section{Acknowledgments}

Authors would like to express their gratitude to the turbine manufacturer for allowing the information and data to be published.

\begin{tabular}{|c|c|}
\hline \multicolumn{2}{|l|}{ Nomenclature } \\
\hline $\mathbf{f}_{h}$ & -hydrodynamic force vector in journal bearing \\
\hline$F(t)$ & excitation vector \\
\hline$[M],[K],[D]$ & mass, stiffness and damping matrices \\
\hline$q$ & complex response vector of the system \\
\hline$\left\{Q_{L}\right\}_{j},\left\{Q_{R}\right\}_{j}$ & $\begin{array}{l}\text { system } j \text {-th complex eigenvectors, left and } \\
\text { right }\end{array}$ \\
\hline$x_{J B}, y_{J B}$ & $\begin{array}{l}\text { cartesian coordinates of a shaft in journal } \\
\text { bearing }\end{array}$ \\
\hline$\lambda_{j}=\lambda_{R}+i \lambda_{j I}$ & $\begin{array}{l}\text { system } \mathrm{j} \text {-th eigenvalue (real and imaginary } \\
\text { parts) }\end{array}$ \\
\hline$\Omega$ & rotational speed $[\mathrm{rad} / \mathrm{s}]$ \\
\hline
\end{tabular}

\section{References}

Chalko T.J., Li D.X., 1993, "Modelling Vibration of a Large TurbineGenerator Set-Effects of Alignment" Proceedings of JSME-ASME International Conference on Power Engineering (ICOPE93) Tokyo, Japan, Sept 12-16 1993, pp. 6.

Chalko, T.J., Li D.X., 1994, Explaining turbine vibration changes due to load variation using numerical model of $650 \mathrm{MW}$ unit. Proceedings of ISROMAC-5, Maui, Hawaii 1994, pp 43-54.

Hashemi, Y., 1983, "Alignment Changes and Their Effects on the Operation and Integrity of Large Turbine Generators: Experience in the CEGB South Eastern Region," Steam and Gas Turbine Foundations and Shaft Alignment-IMeci.E Conference Publications, February, pp. 19-30.

Hori, Y., and Uematsu, R., 1980, "Influence of Misalignment of Support Journal Bearings on Stability of a Multi-Rotor System," Tribology International, Vol. 13, No.5, p. 249.

Krodkiewski, J. M., Parszewski, Z. A., and Anastasiadis, P., 1983, "Effect of Hydrodynamic Bearings on Parametric Rotors Instabilities," Proc. of the Sixth World Congress on Theory of Machines and Mechanisms, New Delhi, India, Dec. 15-20, pp. 1295-1299.

Li, D.X., 1990, "Dynamic Optimization of Multi-Bearing Rotors in Terms of System Configuration Parameters", PhD thesis, Department of Mechanical and Manufacturing Engineering, University of Melbourne, Australia.

Mayes, I.W, Davies W.R.G, 1982, "A Method of Evaluating Sensitivity of a Turbo-generator Shaft Line Vibrational Behavior to Changes in Bearing Coefficients", Proc. IFToMM Rotordynamic Conference, Rome.

Parszewski, Z.A., Chalko, T.J., and Li, D.X., 1988, "Dynamic Optimization of Machine Systems Configuration" Chapter in Structural Optimisation, Kulwer Academic Publishers, pp. 217-224.

Parszewski, Z.A., Chalko, T.J., and Li, D.X., 1988, "Turbogenerator Layout for Optimal Dynamic Response-a study and a case history", IMechE paper C250/88, pp. 427-434.

Parszewski, Z.A., Krodkiewski, J.M., 1986, "Machine Dynamics in Terms of the System Configuration Parameters," Proc. Intern. Conference on Rotor Dynamics, Tokyo, Japan, September 14-17, pp. 239-244.

Webster, E., and Gibson, K.S., 1977, "Turbine Monitoring-Experience in CEGB Southeastern Region," I.Mech.E. Conference on Turbine Monitoring, C243/77.

Wang, L.X., Fang, D.Z., Zhang, M.Y., Lin, J.B., Gu, L., Zhong, T.D., Yang, X.A., Xie, D.P., Luo, Z.H., Xiao, B.Q., Cai, H., and Lin, D.X., 1979, "Mathematics Handbook" (in Chinese), People's Educational Press, Beijing, China. 

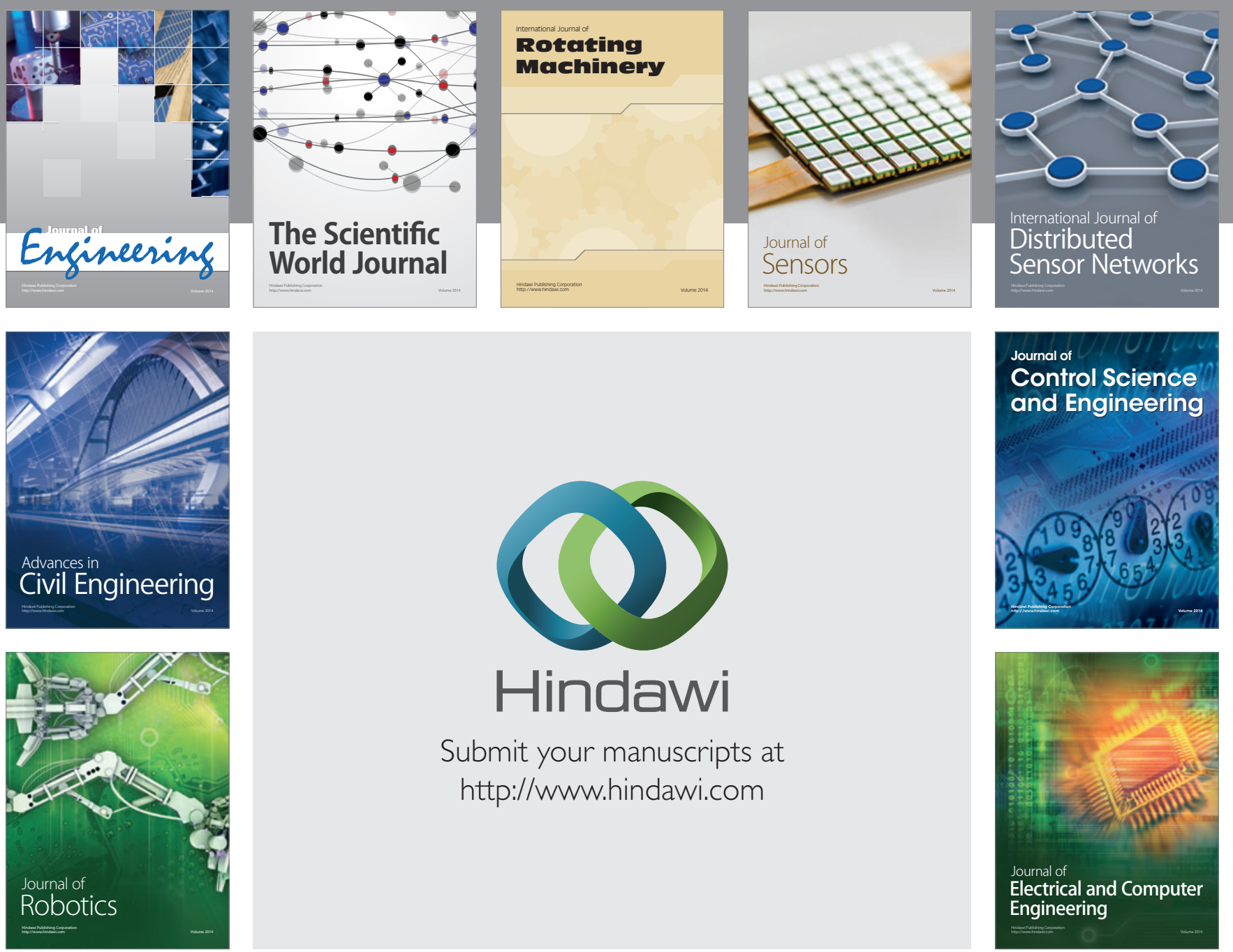

Submit your manuscripts at

http://www.hindawi.com
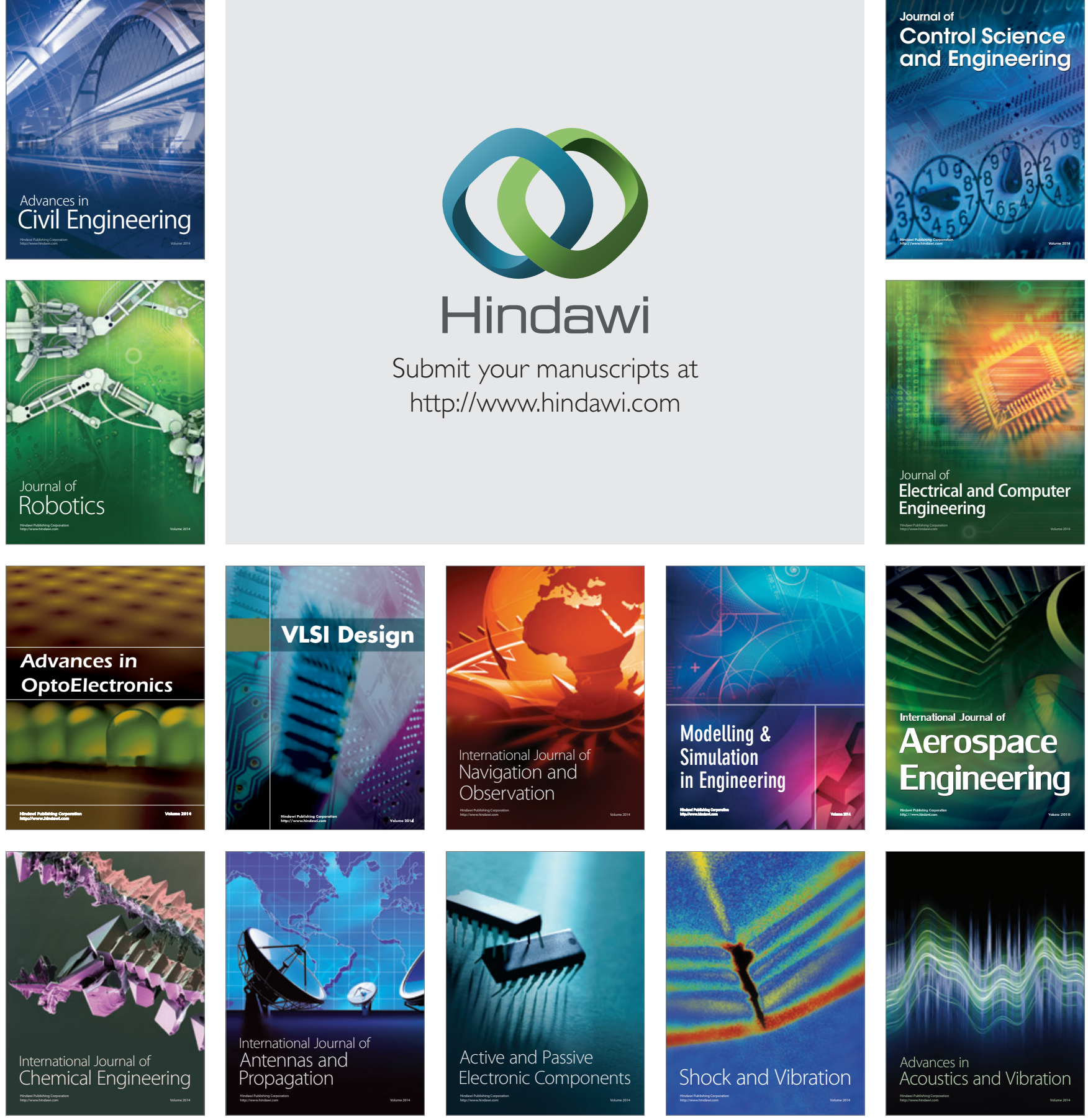